\title{
Globalización, ciudadanía y educación: auge y expansión de los marcos de referencia cosmopolitas, multiculturales y de empoderamiento individual
}

\author{
Francisco O. Ramírez \\ John W. Meyer \\ Christine Min Wotipka
}

Universidad de Stanford

Francisco O. Ramírez es profesor de Educación y Sociología (por cortesía) en la Universidad Stanford. Sus intereses de investigación se centran en las áreas de educación comparativa, sociología política, sociología del desarrollo y género. Su reciente trabajo se enfoca en el aumento y expansión del discurso y organización global de los derechos humanos y en el surgimiento de modelos mundiales de excelencia universitaria y su impacto en universidades alrededor del mundo.

John W. Meyer es profesor emérito de Sociología en la Universidad Stanford.

Christine Min Wotipka es profesora asistente de Educación y Sociología (por cortesía) en la Universidad Stanford. 


\section{Globalización, ciudadanía y educación: auge y expansión de los marcos de referencia cosmopolitas, multiculturales y de empoderamiento individual}

\section{Resumen}

Desde la Segunda Guerra Mundial, la globalización cultural, política y económica ha socavado un primer modelo educacional que solo enfatiza el estado de la nación y la ciudadanía nacional. Más aún, el estudiante debe ser preparado cada vez más para funcionar como titular de derechos y persona responsable en una sociedad global, que se relaciona con otras personas sin reparar en la ciudadanía nacional. Esta sociedad global es considerada cada vez más como legítimamente muy diversa y con un carácter multicultural. Y la diversidad en la sociedad nacional es reconocida hoy como legítima y central. A nivel individual, los estudiantes deben aprender a expresar y respetar todo tipo de valores y materiales culturales únicos.

En este artículo planteamos preguntas en torno a dos cambios centrales. La primera atañe al grado en que los currículos nacionales en ciencias sociales avanzan en la dirección general de la globalización y el multiculturalismo, como corriente opuesta a la conservación de posturas más orientadas a lo nacional. Segundo, analizamos las maneras en que los currículos nacionales resuelven las tensiones entre la construcción de la nación y su ciudadanía y la preparación de los estudiantes como individuos que participan en una sociedad nacional y global diversa.

Proponemos futuros análisis para codificar y analizar los libros de texto de ciencias sociales en unos sesenta países a lo largo de los últimos cincuenta años. Estos estudios darán indicios de tendencias a nivel mundial, regional y nacional en textos. Examinarán factores nacionales y transnacionales que influencian la probabilidad de aumento de los marcos de empoderamiento cosmopolitas, multiculturales e individuales. Se analizará además las maneras en que los currículos de Estudios Sociales procuran resolver las tensiones entre la unidad nacional y la diversidad legítima supranacional y subnacional.

Palabras clave: Globalización, Multiculturalismo, Construcción de la nación, Educación ciudadana, Enseñanza de las ciencias sociales. 


\title{
Globalization, citizenship, and education: the rise and spread of cosmopolitan, multicultural, and individual empowerment frames
}

\begin{abstract}
Summary
Since World War II, cultural, political, and economic globalization have undercut an earlier educational model that only emphasizes the nation state and national citizenship. Increasingly, the student is to be prepared to function as a responsible rights-bearing human person in a global society, relating to people regardless of national citizenship status. This global society is seen as legitimately very diverse and multicultural in character. Diversity within national society is also recognized as legitimate and central. At the individual level students are to learn to express and to respect all sorts of unique values and cultural materials.

In this paper, we raise questions surrounding two relevant core changes. The first is the degree to which national curricula in the social sciences move in the broad direction of globalization and multiculturalism, as opposed to retaining their more nationally oriented postures. Second, we consider the ways in which national curricula resolve the tensions between building the nation and its citizenry and preparing students as individual human participants in a diverse national and global society.

We propose future studies to code and analyze social science textbooks from about sixty countries around the world through the last half-century. These studies will trace worldwide, regional, and national trends in textbook emphases. These studies will examine national and transnational factors that influence the likelihood of the rise and spread of cosmopolitan, multicultural, and individual empowerment frames. These studies will also examine ways in which social studies curricula seek to resolve tensions between national unity and both supra-national and sub-national legitimated diversity.
\end{abstract}

Keywords: Globalization, Multiculturalism, Nation building, Civic education, Social science education. 
a rápida globalización ha impactado profundamente al Estado-nación y sus principales instituciones políticas y educativas. La vida humana se organiza cada vez más a una escala supranacional. La globalización -económica, política y sociocultural- ha problematizado las fronteras y solidaridades nacionales al generar estándares transnacionales de desarrollo nacional y de competencia humana, $y$ al valorar las identidades subnacionales y la diversidad multifacética. Los marcos de referencia cosmopolitas y multiculturales desafían los modelos más viejos de Estado-nación delimitado que privilegiaba una ciudadanía culturalmente homogénea. El Estado-nación continúa siendo central en el ámbito organizacional, pero funciona en un mundo en que el nacionalismo primordial extremo ya no tiene buena reputación. La escolarización masiva, que surgió y se expandió históricamente como un proyecto del Estado-nación, también sufre presión para adaptarse a la globalización y cambiar la visión, el contenido y el énfasis de su educación ciudadana. Se piensa que estos cambios destacarán cada vez más la centralidad del estudiante como persona y sujeto que aprende, y no como mero objeto pasivo de los esfuerzos de socialización. También se cree que implican descripciones de sociedad nacional que reconocen y valoran explícitamente los grupos supranacionales y subnacionales y sus aportes al bienestar tanto del país como del mundo.

Estos cambios de enfoque educativo han sido tanto criticados como apoyados en los EE.UU. y en gran parte del mundo, enfrentando a los nacionalistas tradicionales tanto a los partidarios de "un solo mundo" como a los multiculturalistas. ¿Pero cuál es el alcance, la dirección y la magnitud de los cambios en la educación ciudadana y los pesos relativos de las influencias mundiales y los legados históricos nacionales en la configuración o el impedimento de cambios en los currículos de las ciencias sociales? ¿Avanzamos hacia un mundo de fragmentación política y cultural de las naciones? O, más positivamente, ¿nos dirigimos hacia un mundo más propenso a celebrar la humanidad común y la diversidad local? O más bien, los currículos más tradicionales que hacen hincapié en la ciudadanía en sociedades nacionales unificadas pueden continuar dominando el panorama educativo del mundo (ver las discusiones en Schissler \& Soysal, 2005; y las reseñas en Benavot \& Braslavsky, 2007).

Para tratar este amplio e importante tema se requiere estudiar los cambios en la educación ciudadana a través del análisis de los currículos de ciencias sociales en la educación primaria y secundaria en países alrededor del mundo.

166 I Así, se abordaría las siguientes preguntas: 1) ¿En qué medida el estudiante 
confronta cada vez más imágenes de un mundo supranacional en los currículos de Historia, Geografía, Educación Cívica o Estudios Sociales? ¿La legitimación de la diversidad en aquel mundo socava o relativiza la visión de una identidad nacional propia del estudiante? 2) ¿En qué medida se resta énfasis al propio marco cultural nacional del estudiante -central en una visión curricular más temprana- como marco último de la realidad histórica y geográfica, y de la responsabilidad social y cívica? 3) ¿En qué medida los currículos de ciencias sociales celebran hoy al individuo -diverso a través de dimensiones raciales, étnicas, de género y otras-, como actor legítimo tanto en la sociedad local como en la global? ¿De qué manera esos cambios modifican las anteriores lógicas más centradas en solidaridades nacionales exclusivas? 4) ¿La organización de los currículos privilegia cada vez más el aprendizaje activo y otras actividades más en línea con un foco sobre el estudiante individual como un aprendedor empoderado? Y para todas estas preguntas, 5) ¿qué factores nacionales e internacionales afectan el grado de cambio curricular que se da? Específicamente, 6) ¿en qué medida los cambios en el discurso educativo mundial y los lazos nacionales con (o dependencias de) este discurso predicen cambios en el ámbito nacional?

La comparación de currículos entre una amplia gama de países y en un largo periodo de tiempo complementa las líneas comparativas de investigación y refleja indagaciones psicológicas en la socialización política (Torney-Purta, Barber, Wilkenfeld \& Homana, 2008; Torney-Purta, 2007; Torney-Purta \& Amadeo, 2003; Hahn, 1998). Lógicamente, un estudio macrosociológico de esta naturaleza no aporta información directa sobre la socialización política efectiva de los estudiantes, sino un marco comparativo e histórico muy amplio.

\section{Antecedentes y conceptualización}

Los estudios del auge y expansión de la escolarización masiva insisten en el vínculo de esta con el auge del Estado nacional (Mangan, 1994; Ramírez \& Boli, 1987; Meyer, Ramírez, Rubinson \& Boli, 1977; Meyer, Ramírez, Rubinson \& Soysal, 1992). La escolarización masiva fue diseñada tanto para formar el carácter nacional (Tyack, 1966) como para transformar las masas en ciudadanos (Bendix, 1964). Se imaginó a los ciudadanos bien socializados como el cimiento de Estados-naciones más productivos y competitivos (Schudson, 1998). La expansión del sistema Estado-nación en los siglos XIX y XX desencadenó la expansión de la educación masiva. Básicamente en todas partes, la 
aspiración y realidad de una educación universal, obligatoria y estandarizada prendió (Meyer et al., 1992). A medida que se desarrollaba la educación masiva, los viejos lazos curriculares con las autoridades religiosas fueron subordinados a los énfasis nacionalistas emergentes. El foco sobre la lengua nacional se volvió central: por ejemplo, Francia cambió de un país políglota a uno con una lengua nacional unificada impuesta a través del sistema de escolarización masiva (Weber, 1976). En efecto, las lenguas nacionales se volvieron el elemento central del currículo de la escuela primaria (Benavot, Cha, Kamens, Meyer \& Wong, 1991); las lenguas clásicas y locales tendieron a desaparecer (Cha, 1991). Asimismo, la Historia, Geografía, y más tarde los Estudios Cívicos y Sociales aparecieron haciendo fuerte hincapié en el Estado nacional, y la cultura y la sociedad nacionales (Wong, 1991; Benavot et al., 1991). La escolarización masiva se volvió en casi todas partes una creatura del Estado-nación, y reflejó y promovió el nacionalismo (para discusiones sobre esta tendencia respecto a la enseñanza de Historia en los EE.UU., ver FitzGerald, 1979; Moreau, 2003).

La Segunda Guerra Mundial, su secuela y las crisis circundantes del periodo marcaron un cambio de dirección del eslabonamiento entre educación masiva y Estado-nación, un cambio cuyo análisis empírico es el foco central de nuestra propuesta de proyecto. La educación masiva siguió siendo controlada a nivel organizacional por el Estado nacional y en consonancia con la sociedad nacional. Pero cada vez más la legitimidad del nacionalismo y la sensibilidad de los currículos fuertemente nacionalistas fueron puestos en duda (Kaplan, 2006). Hubo un creciente sentido de que muchos males y peligros del mundo eran provocados a la larga por nacionalismos desenfrenados y por los conflictos nacionalistas que estos generaban (UNESCO, 1974). Se consideró asimismo que un nacionalismo exclusivista engendraba intolerancia y discriminación contra grupos subnacionales. Ambas líneas de pensamiento favorecieron estándares internacionales de progreso y justicia que darían forma al Estado-nación y a su ciudadanía. Estos estándares y sus portadores organizacionales e intérpretes profesionales constituyen lo que se ha denominado sociedad mundial (Meyer, Boli, Thomas \& Ramírez, 1997) o globalización cultural (Robertson, 1992). El sistema de las Naciones Unidas, por más frágil que sea, ha sido un foco organizacional central de la reforma educativa diseñada para promover marcos más cosmopolitas y multiculturales globales. La nueva UNESCO desempeñó un papel central en la promoción de la internacionalización del currículo educativo. Este esfuerzo global por cambiar la enseñanza de las ciencias sociales condujo a la formación del reformista Instituto Georg Eckert en Braunschweig, Alemania. 
La desnacionalización curricular constó de tres dimensiones: un marco de referencia cosmopolita global, una perspectiva multicultural y un énfasis en la persona humana (incluido el estudiante) como sujeto con un intenso grado de derechos y de autonomía. El primer elemento es un enfoque curricular en instruir al estudiante para que vea una sociedad mundial legítima que en principio debería involucrar estándares de progreso y justicia comunes que una diversidad de pueblos acepta. El segundo elemento comprende el desarrollo de una perspectiva sobre la diversidad, antes que una unidad adocenada de la sociedad nacional misma. Es decir que la sociedad nacional es retratada como una comunidad humana diferenciada antes que como una estructura solidaria liderada por las élites del Estado-nación (Banks, 2004; Banks \& Lynch, 1986). Finalmente, el currículo desnacionalizado pone de relieve a la persona individual antes que a la comunidad nacional como unidad ontológica central de la nueva sociedad mundial (Meyer, 2007). El individuo está en el centro de las teorías que resaltan tanto el potencial humano como los derechos humanos (Suárez, 2007a, 2006b; Ramírez, 2006a, 2006b; Ramírez \& Moon, 2008). Surgen nociones más cabales de ciudadanía, que resaltan sus raíces globales y sus compromisos de participación en vez de derechos estrechos basados en el Estado y las prácticas mínimas (McLaughlin, 1992; Kennedy, Hahn \& Lee, 2008).

La decadencia del nacionalismo curricular es evidente en el cambio de la educación masiva mundial a largo plazo de una instrucción en historia (supuestamente más nacionalista) a una instrucción en una vena más difusa de estudios cívicos o sociales (Rauner, 1998; Wong, 1991; para una crítica de este cambio en el contexto estadounidense ver Ravitch, 1987). Además, la instrucción misma de Historia se ha alejado del Estado nacional y sus élites políticas y militares como punto central hacia una concepción de la sociedad más anclada en las ciencias sociales, en la que se hace hincapié en asuntos sociales y culturales y se incluye grupos antes excluidos, como las mujeres y las minorías (ver los artículos en Schisler \& Soysal, 2005; Soysal \& Wong, 2007; Dierkes, 2005; Frank, Wong, Meyer \& Ramírez, 2000; Skrentny, 2002; Skutnabb-Kangas \& Cummins, 1988). Por ejemplo, el que los niños escolares identifiquen a los afroamericanos entre las figuras más importantes de la historia estadounidense sugiere un considerable cambio en el énfasis curricular y su influencia (Wineburg \& Monte-Sano, 2008).

Estos cambios curriculares producen tensiones con las nociones clásicas de ciudadanía e instrucción cívica. Las nuevas concepciones globalizadas de la sociedad mundial y los derechos humanos se enraízan en la tradición de la ley natural, y no en las tradiciones de la ley positiva, ni en las estructuras de gobierno cívico y ni en los procesos electorales. Los procedimientos exactos 
mediante los cuales se elige funcionarios y el carácter exacto de sus deberes pueden hoy parecer menos importantes que los estándares considerados universales de los derechos humanos a la participación y a la protección. Se espera que el estudiante aprenda que todas las personas, antes que todos los ciudadanos, poseen los principales derechos y poderes (Soysal, 1994). El auge de la nueva escolarización emancipada para una "ciudadanía flexible" (Ong, 1999) no significa que las antiguas funciones de la escolarización para la ciudadanía desaparezcan. Por cierto, los críticos insisten en que se ha socavado nociones de patriotismo y ciudadanía en una sociedad nacional histórica profundamente significativa y que ellas deben conservarse (Ravitch, 1987; Huntington, 2004). O bien críticos más globalmente orientados consideran que la enseñanza de las ciencias sociales se está quedando empantanada en un pasado nacionalista (Ward, 2006; Loewen, 1995).

¿De qué manera exactamente las presiones internacionales y los legados nacionales aportan potenciales inconsistencias a los desarrollos curriculares en la educación ciudadana? ¿ ¿l estudiante tiene que aprender que la sociedad nacional es marcadamente diferente de la sociedad global? ¿ $O$ que la sociedad nacional es una instancia de estándares y normas globales compartidas respecto al progreso y la justicia? Avanzar en esta última dirección puede requerir una revisión considerable del saber histórico recibido. Es posible que los traicioneros enemigos y las gloriosas victorias de ayer tengan que ser reconstituidos en términos menos patrioteros. Esta orientación puede resultar más fácil para países más desarrollados y más democráticos (Council of Europe, 2007a, 2007b; Eurydice, 2005; Torney-Purta \& Amadeo, 2004) y más difícil para aquellos que enfrentan una historia inmediata más agitada. Los ex países comunistas pueden ser excepcionalmente dependientes del acatamiento de estándares externos (por ejemplo, los de la Unión Europea) pero aún precisan mucha actividad clásica de construcción de una nación. Las sociedades postconflicto enfrentan el mismo dilema (sobre Sudáfrica, ver Cárdenas, 2007 y sobre Irlanda del Norte, Smith, 2003). Los países nuevos, periféricos y plagados de conflictos buscan resolver las tensiones entre la construcción de una nación integrada y la adaptación a estándares mundiales de maneras nunca antes vistas.

\section{Argumentos respecto de los cambios en educación ciudadana}

Presentamos nuestras líneas de razonamiento en diversos planos. A nivel 170 I mundial, sostenemos que el discurso educativo, y las misiones de las organi- 
zaciones, hacen hincapié cada vez más en la importancia de los currículos de ciencias sociales centrados en la sociedad supranacional y la legitimidad de sus diversos grupos; en la sociedad nacional como comunidad social diversa o multicultural, antes que en una estructura político-militar unitaria; y en los derechos y la capacidad de los individuos con sus gustos y opciones diversas, incluidos el empoderamiento del estudiante y del niño. Estas metas requieren el amenguamiento del énfasis sobre los aspectos problemáticos de las sociedades y los Estados nacionales. Esto quiere decir que las doctrinas educativas a nivel mundial se centren cada vez más en las similitudes de todas las sociedades nacionales, y den un dramático énfasis a los rasgos benignos antes que a los oposicionistas y conflictivos de estas sociedades.

En el ámbito nacional, sostenemos que los currículos deben resaltar cada vez más la historia, la geografía y la sociedad supranacionales y los acuerdos civiles y políticos; la diversidad nacional, subnacional y local, y las estructuras sociales o económicas antes que las militares y políticas; y los derechos y capacidades de los individuos (incluyendo de manera prominente a los niños y a los estudiantes) como personas humanas antes que como miembros ciudadanos de un sistema de gobierno local o nacional. Tales cambios seguramente involucrarán el atenuamiento de los aspectos más dramáticos, oposicionistas y conflictivos del pasado nacional. En efecto, esperamos que el cambio de los currículos tienda a amenguar el énfasis en el Estado nacional y sus élites políticas y militares como elementos centrales de la sociedad (respecto a los cambios en China y Taiwán, ver Mao, 1995; Frank, Wong, Meyer \& Ramírez, 2000). Es probable que se retire el énfasis a los aspectos distintivos de la ciudadanía nacional y de los procesos cívicos frente a concepciones de los derechos humanos como naturales y la expansión del potencial humano como clave para el progreso nacional general. Así, una solución para los conflictos potenciales entre un énfasis curricular en la integración y en la unidad nacional y un énfasis general en la sociedad global es reconfigurar el modelo descrito de la sociedad nacional misma. Si la sociedad y la historia nacionales son reinterpretadas como fundamentalmente en consonancia con las normas de un mundo globalizado, el conflicto potencial entre las lealtades ciudadanas y las lealtades humanas más amplias desaparece. Desde luego que esta transformación, dadas las similares historias sangrientas, requiere una verdadera reconstrucción curricular: si la guerra es generalmente un error, entonces se requiere un nuevo conjunto de héroes nacionales.

Además, al estar los temas del caso fuertemente vinculados con la globalización moderna y los estándares educativos globalizados, sostenemos que las tendencias señaladas antes impactan con mayor fuerza en los currículos 
de sociedades nacionales expuestas más directamente a las normas mundiales. Entre estas se encuentran las sociedades nacionales desarrolladas cuyos sistemas educativos y élites están altamente interpenetrados con los modelos mundiales preferidos. Pero los vínculos con los patrones educativos mundiales pueden ser especialmente fuertes en países post conflicto o en transición muy dependientes de los vínculos con Europa y el mundo. Por otro lado, esos países están especialmente presionados a construir naciones y mitos de nacionalidad, de manera que puedan desplegar combinaciones diferenciadas de compromisos tanto nacionalistas como supranacionalistas respecto a los derechos humanos y el orden mundial (o europeo).

Las comparaciones por toda Norteamérica revelan las siguientes diferencias. El multiculturalismo e internacionalismo canadiense produce currículos diversos en las provincias, pero generalmente con una atención global alta y un bajo foco nacionalista. Los currículos de EE.UU. pueden estar más unificados en los estados y más enfocados en la sociedad nacional, pero la tradición individualista conduce a poner de relieve los derechos y la participación individuales antes que las estructuras cívicas (Hahn, 1999) (respecto a las tensiones entre individualismo y multiculturalismo, ver Olneck, 1990, 1993; respecto a un esfuerzo de reconciliación teórica, ver también Reich, 2002). Los currículos mexicanos, enfrentados a los problemas de construcción de la nación y las deficiencias en el proceso de descentralización, conservan posturas más nacionalistas, pero despliegan un alto compromiso simbólico con las normas de la sociedad global aunque pueden tener un bajo centramiento en el estudiante y en el empoderamiento personal (Levinson, 2004).

Por último, a nivel de libro de texto, sostenemos que es más probable que se encuentren los marcos cosmopolitas, multiculturales y de empoderamiento personal en aquellos libros de texto recientemente publicados, en los de Estudios Sociales antes que en los de Historia, y en aquellos que despliegan una pedagogía más centrada en el estudiante. Estas expectativas proceden de los resultados de nuestro estudio de derechos humanos en libros de texto (Meyer, Bromley \& Ramírez, 2008).

\section{Agendas de investigación futura}

Para comprender plenamente los temas antes delineados se requeriría un proyecto de investigación diseñado para permitirnos a) describir las tendencias

172 I nacionales en los énfasis curriculares en educación ciudadana y analizar las 
variaciones comparadas entre países en los niveles de cosmopolitismo, multiculturalismo y empoderamiento personal en los libros de texto de ciencias sociales y b) seguirle la pista a los énfasis organizacionales y discursivos sobre educación ciudadana a nivel mundial. En concreto, este esfuerzo implicaría analizar los libros de texto de ciencias sociales de unos sesenta países en múltiples período de tiempo a lo largo de toda la postguerra. La tarea analítica sería evaluar en qué grado los perfiles en educación ciudadana de un país reflejan características de dicho país (por ejemplo, tipo de régimen o nivel de desarrollo) y legados históricos (por ejemplo, patrimonio cultural, edad del país) frente a los vínculos del país con los nuevos énfasis mundiales respecto a la educación ciudadana. Presentamos aquí lo que este proyecto implicaría en mayor profundidad con estudios de caso cualitativos más detallados de algunos currículos nacionales especialmente relevantes.

\section{Parte I: el estudio cuantitativo internacional de libros de texto}

A fin de captar cambios de largo plazo, se recopilará datos recogidos de libros de texto de toda secundaria retrocediendo hasta 1955, cuando surgieron las concepciones globalizadas modernas de la sociedad orientada hacia el progreso y de sus miembros individuales empoderados. El plan de codificación cubrirá las dimensiones y los campos generales de la enseñanza de Historia, Cívica, Geografía y Ciencias Sociales discutida antes. Además del Instituto Georg Eckert, el cual posee de lejos la mejor colección de libros de texto de ciencias sociales del mundo, con énfasis en el período a partir de la Segunda Guerra Mundial, ${ }^{1}$ también se accederá a otras colecciones de libros de texto. Dos de estas colecciones tienen especial interés: El Centro de Investigación Manes, de la Universidad Nacional de Educación a Distancia, en Madrid, que se especializa en manuales escolares del mundo hispánico, y el Centro Japonés de Investigación de Libros de Texto, en Tokio, dedicado a los currículos y libros de texto asiáticos.

\section{Parte II: el nivel mundial}

Nuestro proyecto evaluaría, empleando análisis cualitativos y cuantitativos, los cambiantes énfasis mundiales en la educación masiva de las ciencias sociales

1 Fue fundado para promover reformas curriculares que amengüen el nacionalismo en la educación, considerado una causa de la Segunda Guerra Mundial. 
desde la Segunda Guerra Mundial, buscando indicadores de marcos cosmopolitas, multiculturales y de empoderamiento individual. En cuanto a las organizaciones, la meta es seguirle la pista al auge de las asociaciones gubernamentales y no gubernamentales mundiales dedicadas al cambio educativo, y las misiones adoptadas por estas (ver las discusiones generales en Boli \& Thomas, 1999; y usos específicos en Chabbott, 2002; y Mundy \& Murphy, 2001). ¿En qué medida estas asociaciones se centran en la enseñanza de las ciencias sociales, y en qué medida conciben que los propósitos centrales de dicha enseñanza son las reformas globalizadoras?

En cuanto a lo discursivo, le seguiremos la pista a la evolución de la atención profesional dirigida a la reconstrucción de la enseñanza de las ciencias sociales a lo largo de las líneas de la globalización. Esto implica seguir las largas series de conferencias patrocinadas por la Oficina Internacional de Educación, y de manera más general por la UNESCO. También es importante seguir la trayectoria del auge de los focos de las pruebas internacionales dirigidos a la enseñanza de las ciencias sociales, especialmente los estudios de la Asociación Internacional para la Evaluación del Rendimiento Educativo (IEA, por su sigla en inglés) (Torney-Porta, Lehmann, Oswald \& Schulz, 2001; Torney, Oppenheim \& Farnen, 1975), para ver qué tipos de nuevas dimensiones de énfasis parecen importantes en sus agendas. También es necesario rastrear los intercambios internacionales en las profesiones educativas más directamente involucradas. Proponemos seguir el discurso de la UNESCO y la larga serie de esfuerzos del Instituto Georg Eckert.

La meta es mapear los desarrollos educativos mundiales y ver en qué medida las tendencias y variaciones de los países están asociadas con estos desarrollos. Suponemos que los desarrollos discursivos y organizacionales a nivel mundial que enfatizan lo que constituye un buen ciudadano en una buena sociedad influirán en la educación ciudadana y la dirigirán hacia marcos más cosmopolitas, más multiculturales y de mayor empoderamiento de las personas. Esta influencia será directa, como en los impactos directos que siguen a conferencias cruciales e, indirectamente, a través de los vínculos nacionales con organizaciones y profesiones internacionales y sus estándares de educación ciudadana. Además asumimos que esta influencia mundial estará mediada por los legados históricos y los perfiles de los países, lo cual hará que la adopción temprana de sus estándares sea más probable en algunos países. La investigación que proponemos analizará estos supuestos y aguzará nuestra comprensión de la relación entre globalización, ciudadanía 174 I y educación. 


\section{Parte III: estudios de caso cualitativos}

Para comprender mejor los procesos de cambio curricular de las ciencias sociales en el periodo moderno se requeriría un conjunto de estudios de caso cualitativos. Estos estudios seguirían los cambios en los casos de particular interés. Analizarían los libros de textos y los syllabi, y comprenderían entrevistas con personas encargadas de formular políticas vinculadas a y con conocimientos sobre el cambio curricular.

Se podría realizar comparaciones por toda Norteamérica rastreando los currículos de ciencias sociales a lo largo de todo el periodo de la postguerra en tres provincias canadienses (Ontario, Quebec y Columbia Británica), tres estados de EE. UU. (Nueva York, Texas y California) y tres estados mexicanos (Distrito Federal, Chiapas y Quintana Roo). La idea es ver cómo los diversos contextos políticos afectan la transformación global de los currículos. Canadá es reconocido por su internacionalismo y multiculturalismo, incluida su diversidad interna bien legitimada. Los Estados Unidos son reconocidos por su foco sobre la unidad nacional simbólica, y también por su foco sobre el individualismo expandido. Se considera que México ha tenido problemas históricos clásicos en la construcción de la nación como identidad nacional, en parte en oposición a su pasado colonial y en parte en reacción a sus lazos con EE.UU.

Los ex países comunistas -y en especial los países de la ex-Unión Soviéticaenfrentan los clásicos problemas de la construcción de la nación frente a formidables telones de fondo (Silova, 2006). Pero ellos también sufren una extrema presión del mundo (y de manera concreta de la Europa a la que desean unirse) para ser cosmopolitas. Con un centro en el periodo post 1985, dos estudios de caso de países de Europa del Este, y dos de ex-repúblicas de la Unión Soviética, revelarían cómo intentan resolver las cuestiones aquí mencionadas.

En el caso de los países postconflicto, la historia nacional no es atractiva pero sí problemática, y puede ser que la globalización dramática (con sus énfasis sobre la diversidad humana y los derechos humanos legítimos) sea más una solución que un problema (Cole, 2007). Los estudios de caso de dos sociedades postconflicto en esta situación -Irlanda del Norte y Sudáfrica- nos permitirían ver cómo reconstruyen las visiones de sociedad nacional, local y global.

\section{Conclusiones}

Este estudio plantea preguntas respecto al alcance, dirección y magnitud de los cambios en la educación ciudadana y los pesos relativos de las influencias 
mundiales y los legados históricos nacionales en configurar o impedir cambios en los currículos de las ciencias sociales. Esperamos dirigir la atención de las investigaciones y las políticas hacia los cambios en marcha en la educación ciudadana, y hacia las variaciones en aquellos cambios en los distintos países y tiempos. Más importante aún, esperamos dirigir la atención hacia el hecho de que esencialmente todos estos temas implicados son temas de nivel mundial, aunque gran parte de las discusiones intelectuales y de políticas son más bien de carácter provincianamente nacional. Anticipamos que el principal impacto tanto teórico como práctico de la educación ciudadana será cambiar la atención hacia un nivel más global.

\section{Referencias Bibliográficas}

Banks, J. (2004). Diversity and Citizenship Education: Global Perspectives. San Francisco: Jossey-Bass.

Banks, J. \& Lynch, J. (1986). Multicultural Education in Western Societies. Westport, CT: Praeger Publishers.

Benavot, A. \& Braslavsky, C. (2007). School Knowledge in Comparative and Historical Perspective: Changing Curricula in Primary and Secondary Education. Hong Kong: University of Hong Kong/Springer.

Benavot, A.; Cha, Y. K.; Kamens, D.; Meyer, J. \& Wong, S. Y. (1991). Knowledge for the Masses: World Models and National Curricula, 1920-1986. American Sociological Review, 56, 85-100.

Bendix, R. (1964). Nation-Building and Citizenship. Nueva York: John Wiley.

Boli, J. \& Thomas, G. (1999). Constructing World Culture: International Nongovernmental Organizations since 1875. Stanford: Stanford University Press.

Cárdenas, S. (2007). Conflict and Compliance: State Responses to International Human Rights Pressure. Filadelfia: University of Pennsylvania Press.

Cha, Y. K. (1991). Effects of the Global System on Language Instruction, 1850-1986. Sociology of Education, 64, 19-32.

Chabbott, C. (2002). Constructing Educational Development: International Development Organizations and the World Conference on Education for All. Nueva York: Falmer Press.

Cole, E. (2007). Teaching the Violent Past: History Education and Reconciliation. Nueva York: Rowman \& Littlefield. 
Council of Europe. (2007a). Education for Democratic Citizenship. Recuperado el 03 de abril de 2009, de http://www.coe.int/t/dg4/education/edc/

Council of Europe. (2007b). European Year of Citizenship through Education. Recuperado el 03 de abril de 2009, de http://www.coe.int/T/E/Com/Files/Themes/ $\mathrm{ECD} /$

Dierkes, J. (2005). The Decline and Rise of the Nation in German History Education. En H. Schissler \& Y. Soysal (Eds), The Nation, Europe, and the World: Textbooks and Curricula In Transition (pp. 82-104). New York: Berghahn.

Eurydice. (2005). Citizenship Education at School in Europe. Bruselas: Eurydice.

FitzGerald, F. (1979). America Revised: History Schoolbooks in the Twentieth Century. Boston: Little, Brown.

Frank, D.; Wong, S. Y.; Meyer, J. \& Ramírez, F. (2000). What Counts as History: A Cross-National and Longitudinal Study of University Curricula. Comparative Education Review, 44, 29-53.

Hahn, C. (1999). Challenges to Civic Education in the United States. En J. TorneyPurta; J. Schwille \& J. Amadeo (Eds.), Civic Education across Countries: Twentyfour National Case Studies From the IEA Civic Education Project (pp. 583-607). Amsterdam: IEA.

Hahn, C. (1998). Becoming Political. Albany: State University of New York Press.

Huntington, S. (2004). Who Are We? The Challenges to America's National Identity. New York: Simon and Schuster.

Kaplan, S. (2006). The Pedagogical State: Education and the Politics of National Culture in Post-1980 Turkey. Stanford: Stanford University Press.

Kennedy, K.; Hahn, C. \& Lee, W. (2008). Constructing Citizenship: Comparing The Views of Students in Australia, Hong Kong, and the United States. Comparative Education Review, 52, 53-91.

Levinson, B. (2004). Hopes and Challenges for the New Civic Education in Mexico: Toward a Democratic Citizen without Adjectives. International Journal of Educational Development, 24, 269-282.

Loewen, J. (1995). Lies My Teacher Told Me: Everything Your American History Textbook Got Wrong. Nueva York: New Press.

Mangan, J.A. (1994). A Significant Social Revolution: Cross-Cultural Aspects of the Evolution of Compulsory Education. Londres: Woburn Press.

Mao, W. (1995). World History in China Mainland and Taiwan Secondary School Textbooks: A Comparative and Historical Study. Disertación doctoral no publicada, Universidad de Stanford, EE.UU. 
McLaughlin, T. (1992). Citizenship, Diversity and Education: A Philosophical Perspective. Journal of Moral Education, 21, 235-250.

Meyer, J. (2007). World Models, National Curricula, and the Centrality of Individual. En A. Benavot \& C. Braslavsky (Eds.), School Knowledge in Comparative and Historical Perspective: Changing Curricula in Primary and Secondary Education (pp. 259-271). Hong Kong: University of Hong Kong/Springer.

Meyer, J.; Boli, J.; Thomas, G. \& Ramírez, F. (1997). World Society and the NationState. American Journal of Sociology, 103, 144-81.

Meyer, J.; Bromley, P \& Ramírez, F. (2008). Human Rights in Social Science Textbooks: Cross-National Analyses, 1975-2006. Documento inédito, Departamento de Sociología/Escuela de Educación, Universidad de Stanford.

Meyer, J. \& Ramírez, F. (2000). The World Institutionalization of Education, En J. Schriewer (Ed.), Discourse Formation in Comparative Education (pp. 111-132). Frankfurt: Peter Lang Publishers.

Meyer, J.; Ramírez, F.; Rubinson, R. \& Boli, J. (1977). The World Educational Revolution, 1950-1970. Sociology of Education, 50, 242-258.

Meyer, J.; Ramírez, F. \& Soysal, Y. (1992). World Expansion of Mass Education, 1870-1980. Sociology of Education, 65,128-149.

Moreau, J. (2003). Schoolbook Nation: Conflicts over American History Textbooks from the Civil War to the Present. Ann Arbor: University of Michigan Press.

Mundy, K. \& Murphy, L. (2001). Transnational Advocacy, Global Civil Society? Emerging Evidence from the Field of Education. Comparative Education Review, 45, 85-126.

Olneck, M. (1993). Terms of Inclusion: Has Multiculturalism Redefined Equality in American Education? American Journal of Education, 101, 234-260.

Olneck, M. (1990). The Recurring Dream: Symbolism and Ideology in Intercultural and Multicultural Education. American Journal of Education, 98, 147-174.

Ong, A. (1999). Flexible Citizenship: The Cultural Logics of Transnationality. Durham: Duke University Press.

Ramírez, F. (2006a). Beyond Achievement and Attainment Studies: Revitalizing a Comparative Sociology of Education. Comparative Education, 42, 1-19.

Ramírez, F. (2006b). From Citizen to Person: Rethinking Education as Incorporation. En D. Baker \& A. Wiseman (Eds.), The Impact of Comparative Education Research on Institutional Theory (pp. 367-388). Amsterdam: Elsevier.

Ramírez, F. \& Boli, J. (1987). The Political Construction of Mass Schooling: European Origins and Worldwide Institutionalization. Sociology of Education, 60, 2-17. 
Ramírez, F. \& Moon, R. (2008). From Citizenship to Human Rights to Human Rights Education. A ser publicado en International Institute for Legal Studies Series. Oñati: Hart Publishers.

Rauner, M. (1998). The Worldwide Globalization of Civics Education Topics. Disertación doctoral no publicada, Universidad de Stanford, EE.UU.

Ravitch, D. (1987). Tot Sociology: On What Happened to History in the Grade Schools. The American Scholar Summer, 343-354.

Reich, R. (2002). Bridging Liberalism and Multiculturalism in American Education. Chicago: University of Chicago Press.

Robertson, R. (1992). Globalization: Social Theory and Global Culture. Londres: Sage.

Schissler, H. \& Soysal, Y. (2005). The Nation, Europe, and the World: Textbooks and Curricula In Transition. Nueva York: Berghahn.

Schudson, M. (1998). The Good Citizen: A History of American Civic Life. Cambridge: Harvard University Press.

Silova, I. (2006). From Sites of Occupation to Symbols of Multiculturalism: Reconceptualizing Minority Education in Post-Soviet Latvia. Charlotte, NC: Information Age Publishing Inc.

Skrentny, J. (2002). The Minority Rights Revolution. Cambridge: The Belknap Press of Harvard University Press.

Skutnabb-Kangas, T. \& Cummins, J. (1988). Minority Education: From Shame to Struggle. Clevedon, Reino Unido: Multilingual Matters.

Smith, A. (2003). Citizenship Education in Northern Ireland: Beyond National Identity? Cambridge Journal of Education, 32 (1), 15-31.

Soysal, Y. (1994). Limits of Citizenship: Migrants and Postnational Membership in Europe. Chicago: University of Chicago Press.

Soysal, Y. \& Wong, S. Y. (2007). Educating Future Citizens in Europe and Asia. En A. Benavot \& C. Braslavsky (Eds.), School Knowledge in Comparative and Historical Perspective: Changing Curricula in Primary and Secondary Education (pp. 73-88). Hong Kong: University of Hong Kong/Springer.

Suárez, D. (2007a). Education Professionals and the Construction of Human Rights Education. Comparative Education Review, 51, 48-70.

Suárez, D. (2007b). Human Rights and Curricular Policy in Latin America and the Caribbean. Comparative Education Review, 51, 329-352.

Torney, J.; Oppenheim, A. \& Farnen, E. (1975). Civic Education in Ten Countries: An Empirical Study. Nueva York: John Wiley and Sons. 
Torney-Purta, J. (2007). Special Issue: Reflections on the IEA Civic Education Study (1995-2005). Citizenship Teaching and Learning 3.Children's Identity \& Citizenship, European Asociation.

Torney-Purta, J. \& Amadeo, J. A. (2004). Strengthening Democracy in the Americas through Civic Education: An Empirical Analysis Highlighting the Views of Students and Teachers. Washington, DC: Organization of American States.

Torney-Purta, J. \& Amadeo, J. A. (2003). A cross-national analysis of political and civic involvement among adolescents. Political Science and Politics, 36, 269-274.

Torney-Purta, J.; Barber, C.; Wilkenfeld, B. \& Homana, G. (2008). Profiles of civic life skills among adolescents: Indicators for researchers, policymakers, and the public. Child Indicators Research, 1, 86-206.

Torney-Purta, J.; Lehmann, R.; Oswald, H. \& Schulz, W. (2001). Citizenship and Education in Twenty-Eight Countries: Civic Knowledge and Engagement at Age Fourteen. Amsterdam: International Association for the Evaluation of Educational Achievement.

Tyack, D. (1966). Forming of the national character: Paradox in the educational thought of the revolutionary generation. Harvard Educational Review, 36, $37-41$.

UNESCO. (1974). Recommendation Concerning Education for International Understanding, Cooperation and Peace, and Education Relating to Human Rights and Fundamental Freedoms. París: UNESCO.

Ward, K. (2006). History in the Making: An Absorbing Look at How American History Has Changed in the Telling over the Last 200 years. Nueva York: New Press.

Weber, E. (1976). Peasants into Frenchmen: The Modernization of Rural France, 1870-1914. Stanford: Stanford University Press.

Wineburg, S. \& Monte-Sano, C. (2008). 'Famous Americans': The Changing Pantheon of American Heroes. Journal of American History, 94, 1186-202.

Wong, S. Y. (1991). The Evolution of Social Science Instruction, 1900-1986: A CrossNational Study. Sociology of Education, 64, 33-47. 